\title{
Primary Topography-Guided LASIK: A Comparative Study Comparing Treating the Manifest versus the Topographic Astigmatism
}

This article was published in the following Dove Press journal: Clinical Ophthalmology

\section{Fayrouz Aboalazayem (1D) Mohamed Hosny (D) \\ Cherif Zaazou \\ Mohamed Anis (iD \\ Ophthalmology Department, Kasr Alainy Faculty of Medicine, Cairo University, Cairo, Egypt}

Correspondence: Fayrouz Aboalazayem Cairo University, 108 Alshaheed Ahmed Hamdy St., Madkour, Cairo, Egypt

Tel +20 I0074I9500

Fax +20223636504

Email fayrouzaboalazaym@gmail.com
Purpose: To investigate if topography-guided laser in situ keratomileusis (LASIK) depending on the topographic astigmatism which is measured using the Topolyzer leads to a better refractive outcome when compared to treatment of the manifest refractive astigmatism in cases of myopic astigmatism.

Methods: This was a prospective non-masked, randomized study (block randomization) of postoperative vision and refraction of patients with myopic astigmatism that had LASIK using Contoura vision software. They were divided into three groups according to the treatment strategy, treating the manifest astigmatism in one group, the topographic astigmatism with compensation for the spherical power in the second group and treating the topographic astigmatism without changing the spherical power in the third group. It was conducted at Kasr Alainy Hospital and Dar Eloyon Hospital.

Results: The postoperative uncorrected distant visual acuity (UDVA) in each group separately was better than the preoperative corrected distant visual acuity (CDVA) $(58 \%(n=35)$ had UDVA better than 20/20 and gained 1 line or more); however, eyes treated with the topographic astigmatism without changing the spherical power showed the statistically best results $(75 \%(n=15)$ had UDVA better than 20/20). The residual anterior corneal astigmatism was lower in this group (the mean 0.47 vs 0.95 and 0.59 ). No significant difference was noted in the residual refractive astigmatism, but it was also the least in that group.

Conclusion: Topography-guided LASIK is a safe and effective ablation profile for treatment of myopic astigmatism. Treating according to the topographic astigmatism shows the best outcome as regards the vision and residual astigmatism.

Keywords: LASIK, Contoura, TMR

\section{Introduction}

The clinically measured refractive astigmatism (RA) and topography-measured anterior corneal astigmatism (ACA) are rarely similar in the magnitude and axis. ${ }^{1}$ The vectorial difference between both is termed the ocular residual astigmatism (ORA). ${ }^{1}$ Corneal higher-order aberrations (HOAs) are thought to contribute to the ORA., ${ }^{2,3}$

According to Motwani hypothesis, corneal higher order aberrations are not included separately in the manifest refraction. ${ }^{4}$ These aberrations sum up to an astigmatic equivalent, which has an amount and axis and adds to the anterior corneal astigmatism to result in the manifest astigmatism. ${ }^{4}$

Topography-guided ablation uses high-resolution corneal topographic maps to generate a customized ablation profile consisting of anterior corneal higher order aberrations 
(HOAs) and an accurate measurement of the anterior corneal astigmatism (ACA). ${ }^{4}$ They address the irregularities of the corneal elevation in an effort to reshape the cornea into an ideal curve and achieve a regular surface postoperatively.

The US Food and Drug Administration (FDA) approved the "WaveLight Contoura topography-guided platform (Alcon Laboratories, Inc., Fort Worth, TX)", for the primary treatment of myopic astigmatism and they used the manifest refraction as the treatment input. ${ }^{5}$

Most of the laser vision correction strategies depends on the manifest refraction in the treatment which depends on the subjective interaction between the clinician and the patient. However data obtained from the Topolyzer (the value and axis of astigmatism) is often different from the manifest refraction.

The current debate is how to deal with patient refraction if there is a difference between manifest refraction and Topolyzer measured astigmatism. Thus, the idea of adjustment of the manifest refraction with topographic data came to mind and the LYRA protocol was introduced. ${ }^{4}$

\section{Patients' Materials and Methods Ethical Committee}

The study received approval from the ethics committee of Kasr Alainy medical school and registered at clinical trial.gov under the title of (Topography-guided LASIK by different protocols for treatment of astigmatism) with an ID NCT03597906. All procedures fulfilled the Declaration of Helsinki. Written informed consent was obtained from each subject undergoing the procedure at the time of the intervention. Informed consent was also taken from each participant, stating that their preoperative and postoperative unidentified data and scans will be used for scientific publication. All medical data are available for 5 years. They are accessible to any researcher upon his request by contacting the author. The regulations in our country state to keep a copy of medical records for 5 years.

\section{Study Design}

This was a prospective, comparative, randomized, nonmasked, interventional study. It was conducted on 60 eyes of 30 patients with myopic astigmatism of 1.5 diopters or more in both eyes who were randomized with stratified randomization (each subject is included in a group). They underwent uncomplicated primary bilateral topography-guided LASIK on the same refractive surgery platform (FS200 femtosecond and EX500 excimer lasers). The study was performed at Kasr Alainy Hospital and Dar Eloyon Hospital by the same surgeon (MH).
Patients with ocular diseases as a history of herpetic eye disease or corneal dystrophy, keratoconus (as evidenced by Placido topography) or contact lens warpage, corneal scaring, severe ocular surface disease, glaucoma, and collagen vascular diseases and patients less than 18 years were excluded from the study.

\section{Image Acquisition}

An average of six corneal topographic images was captured with the WaveLight Topolyzer VARIO (Alcon Laboratories, Inc.) without dilatation in each eye. Criteria for image acceptance included adequate recognition of pupil and mire edge by the software, with minimal mire breaks, absence of missing data (due to shadow from the eyelids or eyelashes), the analyzed area should be greater than $90 \%$ in the $6.5-\mathrm{mm}$ zone. The reproducibility of data as the axis of astigmatism, the keratometry, and the Q value were assessed by comparing the median of absolute deviation variability score below 0.10 maps. Images not meeting the criteria were excluded. The remaining correct images were exported to the Contoura software to generate the ablation profiles.

\section{T-CAT Surgical Planning}

The eyes were divided into three groups:

- Group A [Manifest group]: 20 eyes of 10 patients were treated with the standard manifest refraction, as in Figure 1.

Example: In a patient with manifest refraction $-1.00 /-$ $2.50 \times 130$ and the anterior corneal astigmatism (Topolyzer) $-3.00 \times 120$, the treatment refraction is: $-1.00 /-2.50 \times 130$.

- Group B [Full TMR]: 20 eyes were treated using the topographic astigmatic power and axis and modifying the spherical power to obtain the same spherical equivalent as the manifest refraction. This was done by subtracting half of the difference between topographic astigmatic power and the manifest astigmatic power from the spherical power (topographymodified refraction treatment), as in Figure 2.

Example: In a patient with manifest refraction $-2.00 /-$ $2.00 \times 160$ and the anterior corneal astigmatism (Topolyzer) $-3.00 \times 175$, the treatment refraction is: $-1.50 /-3.00 \times 175$.

- Group C [partial TMR]: 20 eyes were treated with Contoura vision using the topographic astigmatic power 
WaveLight ${ }^{\circ}$ EX500 | Treatment Report

Gender:
ID:

\begin{tabular}{|c|c|c|c|c|c|}
\hline \multicolumn{6}{|c|}{\begin{tabular}{l|l} 
Refraction $-3.25 \mathrm{D}-1.50 \mathrm{D} @ 175^{\circ} / 12.0 \mathrm{~mm}$
\end{tabular}} \\
\hline \multicolumn{6}{|c|}{ Pupil/Medication $6.5 \mathrm{~mm} /$ keines } \\
\hline \multirow{2}{*}{ Pachymetry } & Superior & Temporal & Central & Nasal & Inferior \\
\hline & $650 \mu \mathrm{m}$ & $618 \mu \mathrm{m}$ & $538 \mu \mathrm{m}$ & $617 \mu \mathrm{m}$ & $608 \mu \mathrm{m}$ \\
\hline K1 / Q1 & \multicolumn{5}{|c|}{$43.83 \mathrm{D} @ 175 \% /-.-$} \\
\hline K2 / Q2 & \multicolumn{5}{|c|}{$46.23 \mathrm{D} @ 85^{\circ} /---$} \\
\hline
\end{tabular}

Treatment details

Measured $-0.61 \mathrm{D}-2.72 \mathrm{D} @ 176^{\circ} / 12 \mathrm{~mm}$

Target --- D -- D @ -.- $\% /---\mathrm{mm}$

Correction $-3.25 \mathrm{D}-1.50 \mathrm{D} @ 175^{\circ} / 12 \mathrm{~mm}$

Target Q ---

Optical zone $6.50 \mathrm{~mm}$

Transition zone $1.25 \mathrm{~mm}$

Ablation zone $9.00 \mathrm{~mm}$

Treatment related information

\begin{tabular}{r|l}
$\begin{array}{r}\text { Cyclorotation } \\
\text { (static) }\end{array}$ & $-1.0^{\circ}$ \\
\hline Centration $\mathbf{X} / \mathbf{Y}$ & $-317 \mu \mathrm{m} / 176 \mu \mathrm{m}$ \\
\hline Total duration & $12 \mathrm{~s}$
\end{tabular}

Breaks $0(0 \mathrm{~s})$

ALCON Laboratories, Inc.

6201 S. Freeway

Fort Worth, TX 76134, USA

\section{OD}

Alcon

Figure I Contoura planning of the manifest refraction correction (group A).

and axis and without change in the spherical power (using the same spherical power as the manifest refraction).

Example: In a patient with manifest refraction $-3.00 /-$ $2.00 \times 110$ and the anterior corneal astigmatism (Topolyzer) $-3.00 \times 120$, the treatment refraction is $-3.00 /-3.00 \times 120$.

\section{LASIK Surgical Technique}

All patients underwent the same refractive platform (FS200 femtosecond laser; Alcon Laboratories, Inc.) to create a flap between $90-110 \mu \mathrm{m}$ with diameter $8: 9 \mathrm{~mm}$ oval flaps with side cuts $135^{\circ}$. The flap is then lifted and the EX500 excimer laser (Alcon Laboratories, Inc.) is applied to perform corneal ablation. The target was emmetropia in all eyes. A postoperative regimen of artificial tears, gatifloxacin $0.3 \%$ antibiotics, and prednisolone acetate $1.0 \%$ eye drops was given. ${ }^{6}$

\section{Statistical Analysis}

The patients were examined preoperatively and at 1 day, 1 week, 1 month, and 3 months postoperatively. The data collected included $\log$ MAR uncorrected and corrected distance visual acuity (UDVA and CDVA), manifest cylindrical refraction, manifest spherical equivalent (SEQ), and anterior corneal astigmatism obtained from the Topolyzer. Astigmatism correction was assessed using the Alpins vector analysis method. ${ }^{7}$ We did not consider posterior corneal astigmatism because, as most researchers found, the posterior corneal astigmatism contribution does not exceed $0.5 \mathrm{D}$ in $87 \%$ of the population.

Data were coded and entered using the statistical package for the Social Sciences (SPSS) version 25 (IBM Corp., Armonk, NY, USA). Data were summarized using mean, standard deviation, minimum and maximum in quantitative data and using frequency (count) and relative frequency (percentage) for categorical data. Comparisons between quantitative variables were done using the non-parametric KruskalWallis and Mann-Whitney tests. For comparison of serial measurements within each patient, the non-parametric Wilcoxon signed rank test was used. For comparing categorical data, Chi square $\left(X^{2}\right)$ test was performed. Exact test was used instead when the expected frequency is less than 5 . $P$-values less than 0.05 were considered as statistically significant.

\section{Results}

Out of the 30 patients, 15 were females. Mean age at the time of the operation was $27.5 \pm 5.6$ years, ranging from 19-45 years. The preoperative refractive variables are 
WaveLight ${ }^{\circ}$ EX500 | Treatment Report

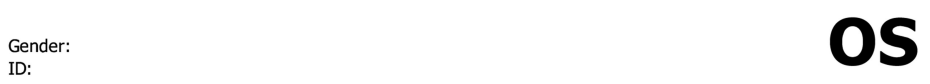

Refractive \& Corneal details

\begin{tabular}{|c|c|c|c|c|c|}
\hline Refraction & $-5.00 \mathrm{D}-2.00 \mathrm{D}$ & $12.0 \mathrm{~mm}$ & & & \\
\hline Pupil/Medication & $6.5 \mathrm{~mm} /$ keines & & & & \\
\hline & Superior & Temporal & Central & Nasal & Inferior \\
\hline raciiy & $721 \mu \mathrm{m}$ & $675 \mu \mathrm{m}$ & $603 \mu \mathrm{m}$ & $685 \mu \mathrm{m}$ & $667 \mu \mathrm{m}$ \\
\hline K1 / Q1 & 43.55 D@ 175 & & & & \\
\hline K2 / Q2 & $45.98 \mathrm{D} @ 85^{\circ}$ & & & & \\
\hline
\end{tabular}

Treatment details

\begin{tabular}{|c|c|c|}
\hline Measured & $-0.65 \mathrm{D}-2.45 \mathrm{D} @ 176^{\circ} / 12 \mathrm{~mm}$ & \\
\hline Target & --- D --- D @ --- ०/ --- mm & \\
\hline Correction & $-4.75 \mathrm{D}-2.50 \mathrm{D} @ 175^{\circ} / 12 \mathrm{~mm}$ & \\
\hline Target Q & -- & Nomogram S 101 \\
\hline Optical zone & $6.50 \mathrm{~mm}$ & Planned flap $120 \mu \mathrm{m}$ \\
\hline Transition zone & $1.25 \mathrm{~mm}$ & Cornea thickness $603 \mu \mathrm{m}$ \\
\hline Ablation zone & $9.00 \mathrm{~mm}$ & Residual stroma $377 \mu \mathrm{m}$ \\
\hline
\end{tabular}

Treatment related information

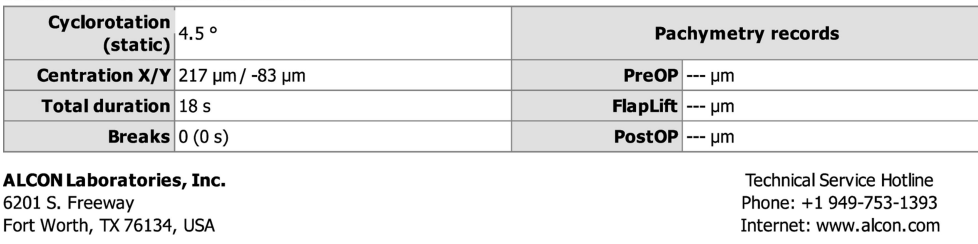

Alcon

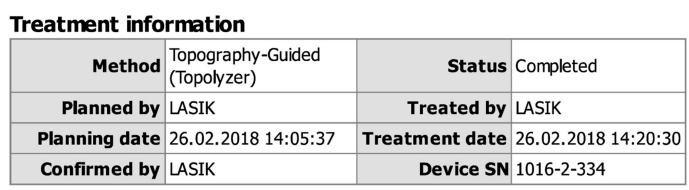

Ablation profile

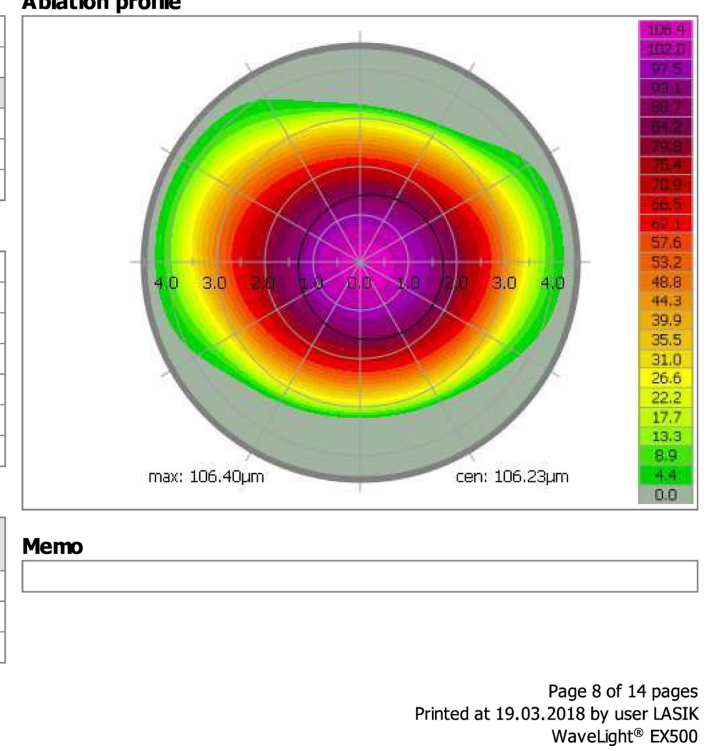

Figure 2 Contoura planning of the full topography modified refraction correction (group B).

reported in Table 1. The postoperative refractive variables are reported in Table 2.

Statistical data were analyzed at 3 months postoperatively showing the following regarding:

\section{Efficacy}

The postoperative UDVA was $-0.06 \pm 0.11 \log$ MAR in the manifest group [A], $-0.05 \pm 0.12 \log$ MAR in the full TMR group [B], and $-0.13 \pm 0.13 \log$ MAR in the partial TMR group [C] (Figure 3A). Cumulative postoperative UDVA in the manifest group was $20 / 20,20 / 25$, and $20 / 30$ in $85 \%$ $(\mathrm{n}=17), 95 \%(\mathrm{n}=19)$, and $100 \%(\mathrm{n}=20)$ of eyes, compared $80 \%(n=16), 95 \%(n=19)$, and $100 \%(n=20)$ in the full TMR group, and $80 \%(\mathrm{n}=16), 100 \%(\mathrm{n}=20)$, and $100 \%(\mathrm{n}=20)$ of eyes in the partial TMR Group (Figure 3B). The mean postoperative UDVA was significantly better than the preoperative CDVA in the three groups (Figure 3C-E) with a $P$-value $<0.05$.

\section{Refractive Accuracy}

Preoperatively and postoperatively, the mean SEQ was $-2.79 \pm 2.21$ and $-0.06 \pm 0.15 \mathrm{D}$ in the manifest group $(P$-value $<0.001)$, compared to $-3.16 \pm 1.89$ and -0.09 $\pm 0.20 \mathrm{D}$ in the full TMR group $(P$-value $<0.001)$ and $-3.07 \pm 1.68$ and $-0.06 \pm 0.16 \mathrm{D}$ in the partial TMR group $(P$-value $<0.001)$. When comparing the postoperative SEQ

Table I Preoperative Refractive Variables

\begin{tabular}{|c|c|c|c|c|c|c|c|c|c|c|c|c|}
\hline \multirow{3}{*}{$\begin{array}{l}\text { Parameter } \\
\text { CDVA [logMAR] }\end{array}$} & \multicolumn{4}{|c|}{ Manifest Group (A) } & \multicolumn{4}{|c|}{ Full TMR (B) } & \multicolumn{4}{|c|}{ Partial TMR (C) } \\
\hline & \multirow{2}{*}{$\begin{array}{l}\text { Mean } \\
0.01\end{array}$} & \multirow{2}{*}{$\begin{array}{l}\text { SD } \\
0.08\end{array}$} & \multicolumn{2}{|c|}{ Range } & \multirow{2}{*}{$\begin{array}{l}\text { Mean } \\
0.04\end{array}$} & \multirow{2}{*}{$\begin{array}{l}\text { SD } \\
0.07\end{array}$} & \multicolumn{2}{|c|}{ Range } & \multirow{2}{*}{$\begin{array}{l}\text { Mean } \\
0.01\end{array}$} & \multirow{2}{*}{$\begin{array}{l}\text { SD } \\
0.06\end{array}$} & \multicolumn{2}{|c|}{ Range } \\
\hline & & & -0.10 & 0.30 & & & -0.10 & 0.10 & & & -0.10 & 0.20 \\
\hline $\mathrm{RA}[\mathrm{D}]$ & -2.33 & 0.60 & -3.00 & -1.50 & -2.75 & 0.90 & -4.50 & -1.50 & -2.20 & 0.85 & -5.25 & -1.50 \\
\hline$A C A[D]$ & 2.47 & 0.78 & 1.00 & 3.57 & 2.97 & 1.09 & 1.00 & 4.75 & 2.31 & 0.65 & 1.25 & 4.50 \\
\hline $\mathrm{SE}[\mathrm{D}]$ & -2.79 & 2.21 & -1.00 & -6.25 & -3.16 & 1.89 & -0.50 & -7.50 & -3.07 & 1.68 & -0.50 & -5.75 \\
\hline
\end{tabular}

Abbreviations: SD, standard deviation; D, diopters; RA, refractive astigmatism; $A C A$, anterior corneal astigmatism; CDVA, corrected distance visual acuity; SE, spherical equivalent. 
Table 2 Postoperative Refractive Variables

\begin{tabular}{|c|c|c|c|c|c|c|c|c|c|c|c|c|c|}
\hline \multirow{3}{*}{$\begin{array}{l}\text { Parameter } \\
\text { UDVA logMAR }\end{array}$} & \multicolumn{4}{|c|}{ Manifest Group (A) } & \multicolumn{4}{|c|}{ Full TMR (B) } & \multicolumn{4}{|c|}{ Partial TMR (C) } & \multirow{3}{*}{$\begin{array}{l}\text { P-value } \\
0.04 I^{*}\end{array}$} \\
\hline & \multirow{2}{*}{$\begin{array}{c}\text { Mean } \\
-0.06\end{array}$} & \multirow{2}{*}{$\begin{array}{l}\text { SD } \\
0.11\end{array}$} & \multicolumn{2}{|c|}{ Range } & \multirow{2}{*}{$\begin{array}{c}\text { Mean } \\
-0.05\end{array}$} & \multirow{2}{*}{$\begin{array}{l}\text { SD } \\
0.12\end{array}$} & \multicolumn{2}{|c|}{ Range } & \multirow{2}{*}{$\begin{array}{c}\text { Mean } \\
-0.13\end{array}$} & \multirow{2}{*}{$\begin{array}{l}\text { SD } \\
0.13\end{array}$} & \multicolumn{2}{|c|}{ Range } & \\
\hline & & & -0.20 & 0.20 & & & -0.20 & 0.20 & & & -0.20 & 0.10 & \\
\hline CDVA logMAR & -0.09 & 0.10 & -0.20 & 0.20 & -0.11 & 0.10 & -0.20 & 0.00 & -0.15 & 0.09 & -0.20 & 0.10 & 0.072 \\
\hline residual RA [D] & -0.23 & 0.33 & -1.00 & 0.00 & -0.25 & 0.29 & -0.75 & 0.00 & -0.16 & 0.31 & -1.00 & 0.00 & 0.541 \\
\hline residual ACA [D] & 0.94 & 0.43 & 0.20 & 1.50 & 0.59 & 0.28 & 0.20 & 1.50 & 0.47 & 0.22 & 0.10 & 0.80 & $0.001 *$ \\
\hline SE [D] & -0.06 & 0.15 & -0.40 & 0.25 & -0.09 & 0.20 & -0.50 & 0.25 & -0.06 & 0.16 & -0.50 & 0.25 & 0.802 \\
\hline Sphere [D] & 0.06 & 0.16 & 0.00 & 0.50 & 0.08 & 0.18 & 0.00 & 0.50 & 0.03 & 0.11 & 0.00 & 0.50 & 0.538 \\
\hline
\end{tabular}

Note: *Statistically significant.

Abbreviations: SD, standard deviation; D, diopters; RA, refractive astigmatism; ACA, anterior corneal astigmatism; CDVA, corrected distance visual acuity; SE, spherical equivalent; UDVA, unaided distance visual acuity.

between the three groups, the difference was not statistically significant ( $P$-value $=0.8)$. About $70 \%$ of eyes $(n=14)$ in each group $\mathrm{A}$ and $\mathrm{C}$ have postoperative spherical equivalent -0.2 to 0 and about $55 \%$ of eyes $(n=11)$ in group B have spherical equivalent -0.2 to 0 (Figure $4 \mathrm{~A}$ ).

\section{The Accuracy of Refractive Astigmatism and Anterior Corneal Astigmatism}

Preoperatively and postoperatively, the mean refractive astigmatism was $-2.33 \pm 0.60$ and $-0.23 \pm 0.33 \mathrm{D}$ in the manifest group ( $P$-value $<0.001)$, compared to $-2.75 \pm 0.90$ and $-0.25 \pm 0.29 \mathrm{D}$ in the full TMR group $(P$-value $<0.001)$ and $-2.20 \pm 0.85$ and $-0.16 \pm 0.31 \mathrm{D}$ in the partial TMR group $(P$-value $<0.001)$. When comparing the postoperative refractive astigmatism between the three groups, the difference was not statistically significant $(P$-value $=0.541)$, but it was the least in the partial TMR group.

As regards the anterior corneal astigmatism, the mean preoperative and postoperative values were $2.47 \pm 0.78$ and $0.94 \pm 0.43 \mathrm{D}$ in the manifest group ( $P$-value $<0.001)$, compared to $2.97 \pm 1.09$ and $0.59 \pm 0.28 \mathrm{D}$ in the full TMR group $(P$-value $<0.001)$ and $2.31 \pm 0.65$ and $0.47 \pm 0.22 \mathrm{D}$ in the partial TMR group $(P$-value $<0.001)$. When comparing the postoperative anterior corneal astigmatism between the three groups, the difference was statistically significant $(P$-value $=0.001)($ Figure 4B).

\section{Cylinder Vector Analysis}

Although the astigmatism treatment predictability was comparable using the three treatment protocols, the R2 between the target induced astigmatism (TIA) and the
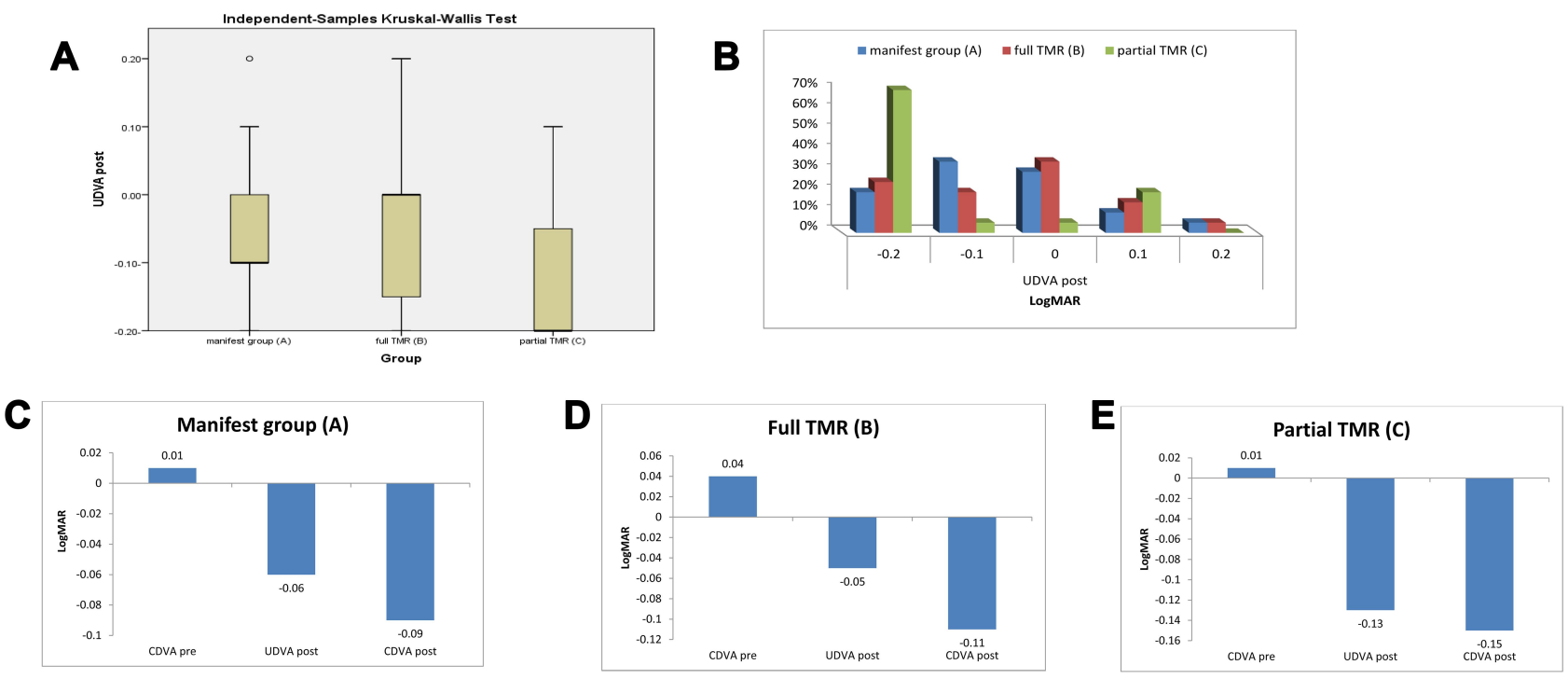

Figure 3 (A) Comparison between the mean postoperative UDVA in the three groups. (B) The cumulative postoperative UDVA in the three groups in logMAR. (C) The mean preoperative and postoperative vision in group (A). (D) The mean preoperative and postoperative vision in group (B). (E) The mean preoperative and postoperative vision in group $(\mathrm{C})$. 
A

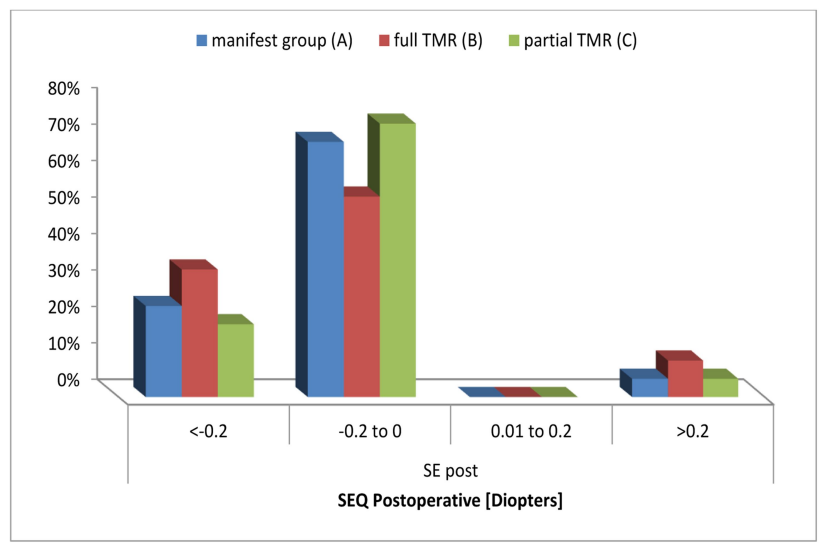

C

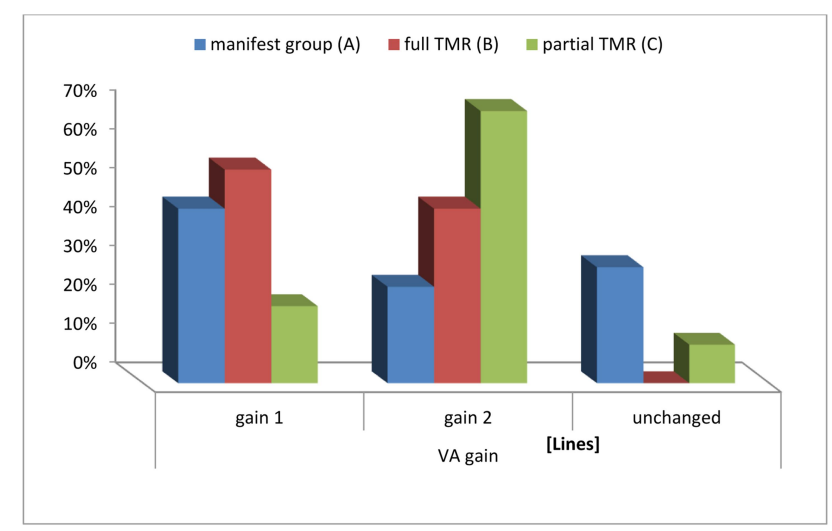

B

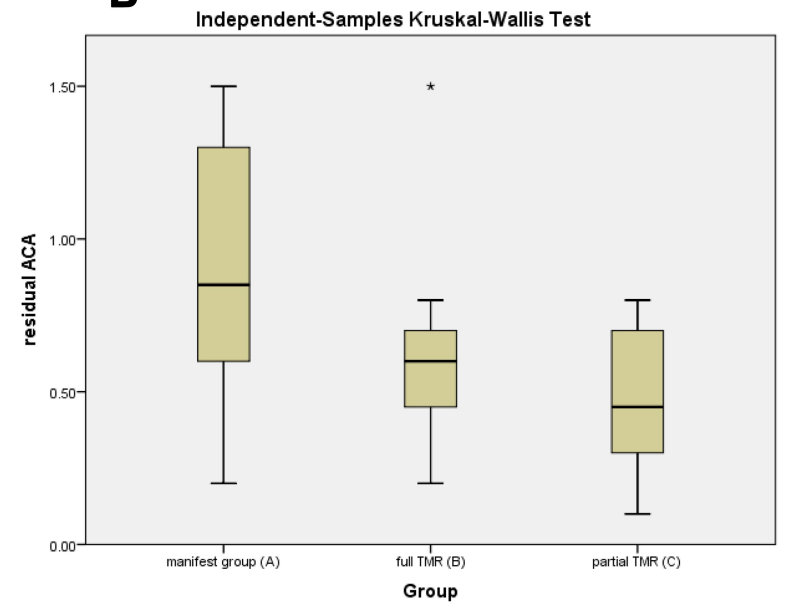

D

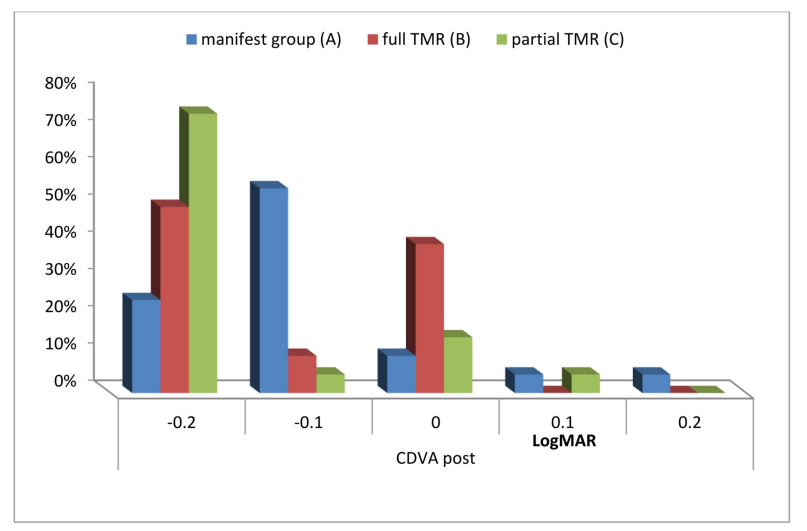

Figure 4 (A) The residual postoperative spherical equivalent in the three groups. (B) The residual anterior corneal astigmatism of the three groups. (C) The postoperative line gain in the three groups. (D) The cumulative postoperative CDVA in the three groups in logMAR.

surgically induced astigmatism (SIA) was close to 0.82 in the manifest group and close to 0.62 in the full TMR group compared to 0.7 in the partial TMR group (Figure 5). The correction index (CI) of eyes was comparable in the three groups and close to 1, as shown in Table 3. Additional Alpins vectors and parameters are reported in Table 3 and graphed as single-angle polar plots in Figure 6.

\section{Safety}

In the three groups, no eye had loss of any line of CDVA (Figure 4D) and close to $70 \%$ of eyes $(n=14)$ in the manifest group gained one line and $25 \%(\mathrm{n}=5)$ gained two lines, while $100 \%$ of eyes $(n=20)$ in the full TMR group gained one line and $45 \%(\mathrm{n}=9)$ gained two lines, in comparison to $90 \%$ of eyes $(\mathrm{n}=18)$ in the partial TMR group which gained one line and $70 \%(\mathrm{n}=14)$ gained two lines (Figure 4C).

\section{Complications}

We did not observe any significant complications, and the patients did not need any enhancements during the follow-up period.

\section{Discussion}

Laser-assisted in situ keratomileusis (LASIK) is one of the most popular Laser vision correction techniques practiced worldwide which has been established over the last two decades as a safe and effective procedure. ${ }^{8}$

Excellent refractive results have been reported when a customized ablation pattern by utilizing topographic data was adopted. ${ }^{9}$

The FDA study demonstrated excellent refractive predictability and uncorrected visual acuity (UCVA) results of topography-guided LASIK, as well as statistically significant subjective visual improvement, although they used 

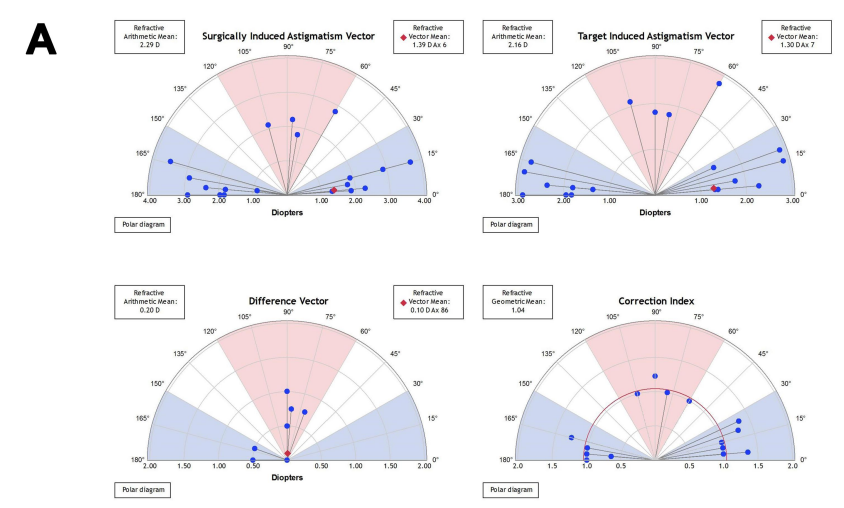

B
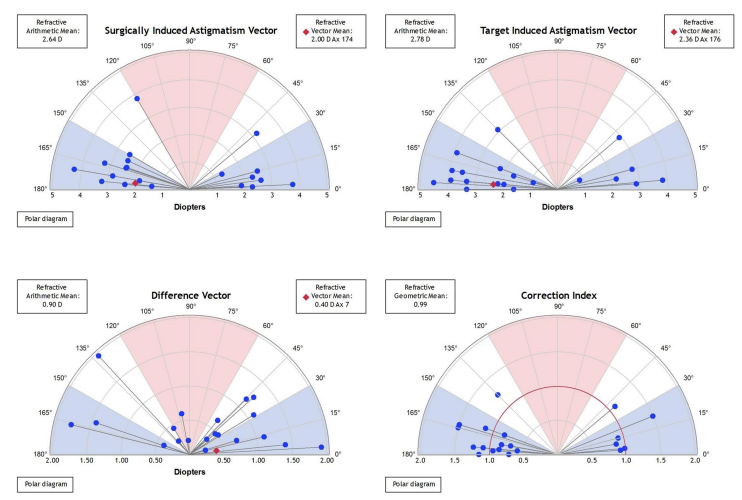

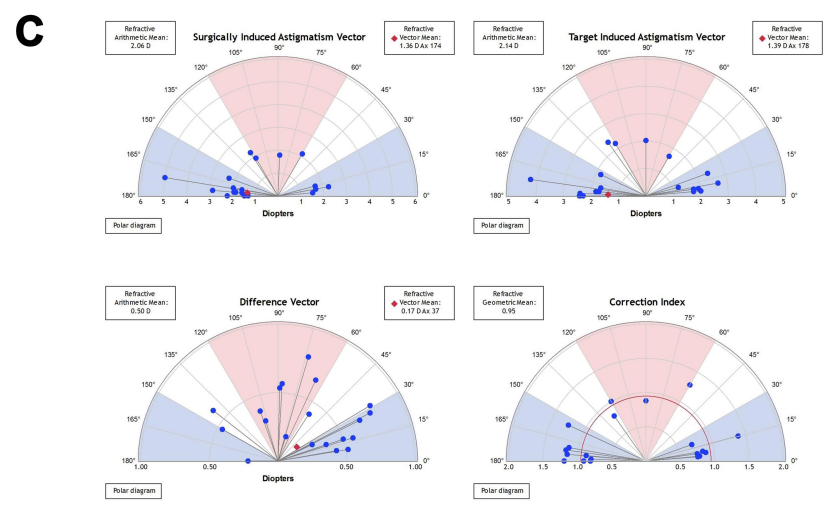

Figure 5 Single-angle polar plots generated using the ASSORT software to illustrate the target induced astigmatism (TIA) vector, the surgically induced astigmatism (SIA) vector, the difference vector (DV), and the correction index $(\mathrm{Cl})$ in the manifest group (A) vs full TMR group (B) and partial TMR group (C). The vector means are plotted as a red diamond.

the manifest refraction in correction. ${ }^{5}$ UCVA results were excellent, with $99 \%$ of the eyes seeing $20 / 32$ or better, $92.7 \%$ seeing $20 / 20$ or better, and $68.8 \%$ seeing $20 / 16$ or better. $^{10}$ No lines of the best-corrected visual acuity (BCVA) were lost, with all patients either maintaining or gaining lines of vision. ${ }^{10}$

These are comparable to our results where we found that more than $80 \%$ of eyes $(n=48)$ achieved $20 / 20$ or better UDVA. About $38 \%$ of eyes $(n=23)$ had $20 / 10$ UDVA. About $46.6 \%$ of eyes $(n=28)$ gained two lines and about $86.6 \%(\mathrm{n}=52)$ gained one line and there was no visual loss of any line. About $66.6 \%$ of eye $(n=40)$ has residual spherical equivalent -0.25 to 0.0 .

The current debate in treating astigmatic patients is whether to use the manifest astigmatism power and axis or using the topographic one.

Table 3 Vector Analysis

\begin{tabular}{|c|c|c|c|c|c|c|c|c|c|c|c|c|}
\hline \multirow{3}{*}{$\begin{array}{l}\text { Parameter } \\
\text { TIA vector [D] }\end{array}$} & \multicolumn{4}{|c|}{ Manifest Group (A) } & \multicolumn{4}{|c|}{ Full TMR (B) } & \multicolumn{4}{|c|}{ Partial TMR (C) } \\
\hline & \multirow{2}{*}{$\begin{array}{l}\text { Mean } \\
2.16\end{array}$} & \multirow{2}{*}{$\begin{array}{l}\text { SD } \\
0.59\end{array}$} & \multicolumn{2}{|c|}{ Range } & \multirow{2}{*}{$\begin{array}{l}\text { Mean } \\
2.78\end{array}$} & \multirow{2}{*}{$\begin{array}{l}\text { SD } \\
1.04\end{array}$} & \multicolumn{2}{|c|}{ Range } & \multirow{2}{*}{$\begin{array}{l}\text { Mean } \\
2.14\end{array}$} & \multirow{2}{*}{$\begin{array}{l}\text { SD } \\
0.61\end{array}$} & \multicolumn{2}{|c|}{ Range } \\
\hline & & & 1.31 & 2.89 & & & 0.87 & 4.52 & & & 1.21 & 4.24 \\
\hline SIA vector [D] & 2.29 & 0.71 & 0.88 & 3.72 & 2.64 & 0.77 & 1.31 & 4.27 & 2.01 & 0.78 & 1.31 & 5.01 \\
\hline DV vector $[D]$ & 0.20 & 0.33 & 0.00 & 1.00 & 0.89 & 0.58 & 0.25 & 1.96 & 0.49 & 0.18 & 0.19 & 0.79 \\
\hline Correction index & 1.04 & 0.16 & 0.64 & 1.36 & 0.99 & 0.27 & 0.59 & 1.50 & 0.95 & 0.21 & 0.70 & 1.39 \\
\hline $\mathrm{AE}$ & -1.22 & 2.55 & -10 & 0 & -2.15 & 8.54 & -14 & 15 & -2.78 & 3.92 & -10 & 5 \\
\hline$\%$ with $\mathrm{AE}$ within $15^{\circ}$ & $100 \%$ & & & & $100 \%$ & & & & & & $100 \%$ & \\
\hline$\%$ with $A E>15^{\circ}$ & 0.0 & & & & 0.0 & & & & & & 0.0 & \\
\hline$\%$ with $A E<-15^{\circ}$ & 0.0 & & & & 0.0 & & & & & & 0.0 & \\
\hline
\end{tabular}

Abbreviations: SD, standard deviation; D, diopters; TIA, target induced astigmatism; SIA, surgically induced astigmatism; DV, difference vector; AE, angle of error. 

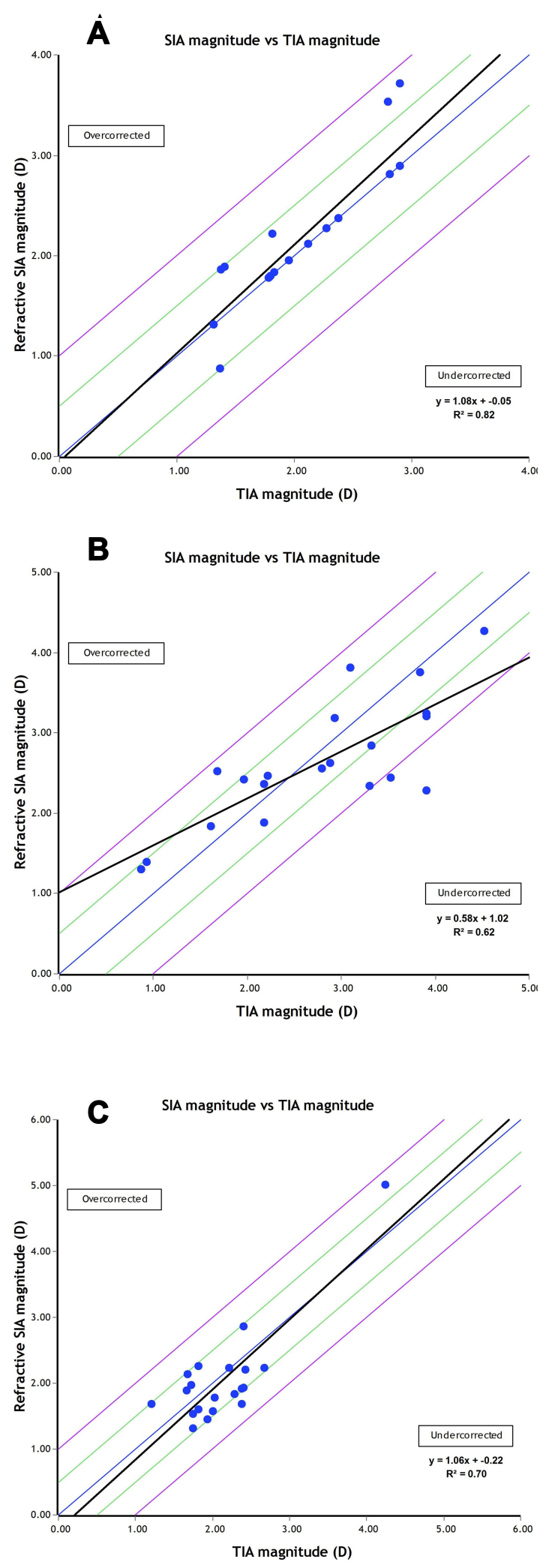

Figure 6 Target induced astigmatism (TIA) vector vs surgically induced astigmatism (SIA) vector (calculated at the corneal plane) of the manifest group (A), the full TMR group (B), and the partial TMR group (C).
Kanellopoulos used the topographic astigmatic power and axis (topography-modified treatment refraction) in the contralateral eye study found that the average postoperative UDVA improved from $20 / 200$ to $20 / 20$ in the manifest group vs 20/16 in the TMR group with more lines gain in the TMR group. ${ }^{9}$

Motwani used the topography measured astigmatism (LYRA protocol) in treatment of 50 astigmatic patients and found that $80.85 \%$ of the patients achieved $20 / 15$ vision, $100 \%$ achieved $20 / 20$ vision, and $6.4 \%$ achieved $20 / 10$ vision with the average difference of astigmatic power from manifest to measured was $0.55 \mathrm{D}$ (with a range of $0-1.69 \mathrm{D}$ ), and the average difference of axis was $14.94^{\circ}$ (with a range of $\left.0^{\circ}-89^{\circ}\right) .^{11}$

In our study we compared three groups, the first one we used the manifest refraction in correction of astigmatism (group A), the second group we used the LYRA protocol (group B) and in the third group used the topographic astigmatism in treatment without change in the spherical power (group C).

We found that about $55 \%$ of eyes in group (A) had UDVA better than $0.00 \operatorname{logMAR}$, while it was $54 \%$ in group (B) and $75 \%$ in group (C).

We also found that in group (A), about $70 \%$ of eyes gained one line and about $25 \%$ of eyes gained two lines, while 30\% remained unchanged. In group (B), about 100\% of eyes gained one line and about $45 \%$ of eyes gained two lines. In group (C), about $90 \%$ of eyes gained one line and about $70 \%$ of eyes gained two lines, while $10 \%$ remained unchanged.

We also found no statistically significant difference between the three groups in the amount of residual manifest refractive astigmatism and the residual spherical equivalent as well.

However there was a statistically significant difference between the three groups in the residual anterior corneal astigmatism, being least in the partial TMR group (C) and highest in the manifest group (A).

This could be attributed to the Motwani ${ }^{4}$ hypothesis that the manifest astigmatism is the vectorial sum of the true corneal astigmatism and the residual corneal astigmatism which results from corneal higher order aberrations, so when using topography-guided ablation in treatment of astigmatism, it neutralizes the higher order aberration so the resultant true corneal astigmatism should be treated not the 
manifest astigmatism, this is what we were applying in group (C). Changing the spherical power when using the topographic astigmatism as in group (B) means that we are not sure of the technology, this explains why we got the best results with the partial TMR group. ${ }^{4}$

In conclusion, adjustment of the power and axis of astigmatism treated using the topography when different from the clinical refraction without change in the spherical power, may offer superior outcomes in topography-guided myopic LASIK. This novel treatment strategy may change the current clinical paradigm of the optimal subjective refraction utilized in laser vision correction.

\section{Disclosure}

The authors have no financial or non-financial conflicts of interest and declare no public or private support.

\section{References}

1. Alpins N. A new method of analyzing vectors for changes in astigmatism. J Cataract Refract Surg. 1993;19:S24-S33.

2. Tan J, Simon D, Mrochen M, et al. Clinical results of topography-based customized ablations for myopia and myopic astigmatism. $J$ Refract Surg. 2012;28:S829-S836. doi:10.3928/ 1081597X-20121005-04
3. Zhou W. Stojanovic A and Utheim TP. Assessment of refractive astigmatism and simulated therapeutic refractive surgery strategies in coma-like-aberrations-dominant corneal optics. Eye Vis (Lond). 2016;3:13.

4. Motwani M. The use of WaveLightContoura to create a uniform cornea: the LYRA protocol. Part 1: the effect of higher-order corneal aberrations on refractive astigmatism. Clin Ophthalmol. 2017;11:897-905.

5. Stulting RD, Fant BS. Results of topography-guided laser in situ keratomileusis custom ablation treatment with a refractive excimer laser. J Cataract Refract Surg. 2016;42:11-18. doi:10.1016/j. jers.2015.08.016

6. Wallerstein A, Gauvin M, Adiguzel E, et al. Clinically significant LASIK flap striae. J Cataract Refract Surg. 2017;43:1523-1533. doi:10.1016/j.jcrs.2017.09.023

7. Alpins N. Astigmatism analysis by the Alpins method. $J$ Cataract Refract Surg. 2001;27:31-49. doi:10.1016/S0886-3350(00)00798-7

8. Lukenda A. Martinović ZK and Kalauz M. Excimer laser correction of hyperopia, hyperopic and mixed astigmatism: past, present, and future. Acta Clin Croat. 2012;51(2):299-304.

9. Kanellopoulos A. Topography-modified refraction (TMR): adjustment of treated cylinder amount and axis to the topography versus standard clinical refraction in myopic topography-guided LASIK. Clin Ophthalmol. 2016;10:2213-2221. doi:10.2147/OPTH.S122345

10. Durrie D. Clinical results of the US T-CAT study. In: Cataract and Refractive Surgery Today. 2011:53-54.

11. Motwani M. The use of wavelight; Contoura to create a uniform cornea: the LYRA protocol. Part 3: the results of 50 treated eyes. Clin Ophthalmol. 2017;11:915-921. doi:10.2147/OPTH.S133841
Clinical Ophthalmology

\section{Publish your work in this journal}

Clinical Ophthalmology is an international, peer-reviewed journal covering all subspecialties within ophthalmology. Key topics include: Optometry; Visual science; Pharmacology and drug therapy in eye diseases; Basic Sciences; Primary and Secondary eye care; Patient Safety and Quality of Care Improvements. This journal is indexed on PubMed
Central and CAS, and is the official journal of The Society of Clinical Ophthalmology (SCO). The manuscript management system is completely online and includes a very quick and fair peer-review system, which is all easy to use. Visit http://www.dovepress.com/ testimonials.php to read real quotes from published authors. 